\title{
EFECTO DE CAMA DE POLLOS, SUBPRODUCTOS DE CEREALES Y CAÑA SOBRE LA FISIOLOGÍA RUMINAL DE OVINOS
}

\author{
EFFECTS OF POULTRY LITTER, CEREAL BYPRODUCTS AND SUGAR CANE BAGASSE \\ ONLAMBRUMINALPHYSIOLOGY
}

\author{
Nouel Borges, G. ${ }^{1 *}$, Hevia Opazo, P. ${ }^{1}$, Velásquez, M. ${ }^{1}$, Espejo Días, M. ${ }^{1}$, Rojas C., J. ${ }^{1}$ \\ y Sánchez B., R. ${ }^{1}$
}

'Unidad de Investigación en Producción Animal. Decanato de Agronomía. Universidad Centroccidental Lisandro Alvarado. Tarabana. Palavecino. CP 3023. Estado Lara. Venezuela. *gustavonouel@ucla.edu.ve

\section{Palabras claVe ADICIONALES}

Consumo. Digestibilidad. Degradabilidad. Pulitura de arroz. Harina de subproductos de maíz.

\section{RESUMEN}

Se determinó el efecto sobre el consumo y digestibilidad aparente de niveles crecientes de cama de pollo (Cp) con harina gruesa de subproductos de maíz $(\mathrm{Hm})$ o pulitura de arroz $(\mathrm{Pa})$ y bagacillo de caña amonificado (40\%, Ba), en raciones para corderos en crecimiento en confinamiento total. Se realizó un experimento en un arreglo factorial $2 \times 4$ en diseño de cuadrado latino con 8 corderos canulados en el rumen, incorporando $\mathrm{Hm}$ o $\mathrm{Pa}(48,39,30$ y $21 \%$, en ambos casos) y $\mathrm{Cp}(11,4 ; 20,4 ; 29,4$ y $38,4 \%)$. Las raciones con $\mathrm{Cp}$ y $\mathrm{Hm}$ fueron más consumidas que las de $\mathrm{Cp}$ y $\mathrm{Pa}$ (10 a $12,1 \%$ del peso metabólico en materia seca (MS), para Hm vs. 7,6 a 9,3\% para $\mathrm{Pa}$ ) y para la materia orgánica (MO) 8,1 a $8,5 \%$ del peso metabólico para $\mathrm{Hm}$ y 5,8 a $6,4 \%$ para $\mathrm{Pa}$; respecto a la digestibilidad aparente (DA) se obtuvo un 34,2 a $43 \%$ de la MS y 67,8 a $67,1 \%$ de la MO para raciones con $\mathrm{Hm}$ vs. 30,5 a $37,2 \%$ de la MS y 65,5 a $66,1 \%$ de la $\mathrm{MO}$ para raciones con $\mathrm{Pa}$. Se apreció que el $\mathrm{pH}$ y el nitrógeno amoniacal, no alcanzaron los valores indicativos de acidosis ni de intoxicación con amoniaco, lo cual se correspondió con valores normales de su fisiología sanguínea para raciones con $\mathrm{Cp}$ y $\mathrm{Hm}$. Los resultados indican que $\mathrm{Hm}$ y $\mathrm{Ba}$ en raciones con $\mathrm{Cp}$ permitieron un consumo y digestibilidad de los nutrientes dentro de rangos normales para corderos, sin riesgos para la salud del animal, lo cual no ocurrió con las raciones que contenían $\mathrm{Pa}$ y $\mathrm{Cp}$.

Recibido: 30-10-07. Aceptado: 2-4-09.

\section{AdDitional KEYWORDS}

Intake. Digestibility. Degradability. Rice polishing. Homini.

\section{SUMMARY}

The effect on intake and digestibility of increasing levels of poultry litter $(C p)$, corn byproduct meal $(\mathrm{Hm})$ or rice polishing $(\mathrm{Pa})$ and sugarcane ammoniated bagasse $(\mathrm{Ba}, 40 \%)$ in rations for growing lambs in total confinement was determined. A factorial arrangement $2 \times 4$ Latin square design, with 8 sheep cannulated in rumen, was used, incorporating $\mathrm{Hm}$ or $\mathrm{Pa}(48,39,30$ and $21 \%$ in both cases) and $\mathrm{Cp}(11.4,20.4,29.4$, and $38.4 \%$ ). Dry matter intake was higher in rations with $\mathrm{Cp}$ and $\mathrm{Hm}$ with respect to $\mathrm{Cp}$ and $\mathrm{Pa}$ (10$12.1 \%$ of metabolic weight for $\mathrm{Hm}$ vs. $7.6-9.3 \%$ for $\mathrm{Pa}$ ) and for organic matter (MO) intake from 8.1 to $8.5 \%$ of metabolic weight for $\mathrm{Hm}$ and $5.8 \%$ to $6.4 \%$ for $\mathrm{Pa}$; the apparent digestibility (DA) of dry matter was $34.2-43 \%$ and $67.8-67.1 \%$ for $\mathrm{MO}$ in rations with $\mathrm{Hm}$ vs. $30.5-37.2 \%$ and $65.5-66.1 \%$ respectively for $\mathrm{Pa}$. The ruminal $\mathrm{pH}$ and amoniacal nitrogen levels, were not indicative of acidosis or ammonia poisoning, which was consistent with normal blood physiology for rations with $\mathrm{Cp}$ and $\mathrm{Hm}$. Results indicate that $\mathrm{Hm}$ and $\mathrm{Ba}$ rations with $\mathrm{Cp}$ allowed consumption and digestibility of nutrients within the normal range for lambs, without risk to animal health, which did not occur with the ratios containing $\mathrm{Pa}$ and $\mathrm{Cp}$.

\section{INTRODUCCIÓN}

Los sistemas de producción con rumian- 
tes permiten el uso de materiales no aprovechables por el hombre, aves o cerdos. En la región centroccidental de Venezuela, el cultivo de caña de azúcar, concentra más del $75 \%$ de la producción y procesamiento nacional; generando 2,74 millones de toneladas de bagacillo (cálculos propios, Ramírez, 2000 y MAC, 1998), que son quemadas en centrales azucareras o acumuladas en sus alrededores. También, la región es la principal productora de cereales del país, con una importante agroindustria procesadora de arroz y maíz (MAC, 1998). Los recursos disponibles y el clima seco, han impulsado el crecimiento del sector avícola, generando abundante producción de excretas, cuyo volumen se estima entre 370000-450000 t/año (cálculos propios, sobre datos del MAC, 1998); la cama de pollos $(\mathrm{Cp})$, puede representar un problema ambiental, al tener que ser eliminada de las unidades de producción inapropiadamente o sin procesamiento. Uno de sus principales usos es como abono orgánico (Fontenot, 1979; Rodrígues et al., 1997) o como alimento para rumiantes.

El bagacillo de caña, subproductos de arroz y maíz, cama de pollos, pueden ser usados en alimentación de ovinos (García, 1980; Chaudhry et al., 1996), con ventajas evidentes en el aprovechamiento de los recursos y reducción del impacto ambiental de estas industrias, favoreciendo un suministro uniforme de alimentos y mejorando la eficiencia productiva de rebaños, con mayores ingresos a los productores. La harina gruesa de subproductos de maíz (Hm) es una materia prima de uso casi exclusivo en rumiantes, por su alto contenido de fibra (hemicelulosa y celulosa), con cantidades de proteínas que varían entre 11 y $14 \%$ (NRC, 1985; Hernández et al., 1999). La Pa (pulitura, afrecho de arroz, rice bran o polishing) representa cerca del $10 \%$ del peso del arroz paddy (Choct, 1997). La Pa de arroz posee $\mathrm{PB} 14,4 \pm 0,4 \%$, FDA (fibra insoluble en detergente ácido) $10,4 \pm 1,4 \%$ y FDN (fibra insoluble en detergente neutro)
$22,0 \pm 2,5 \%$ (DePeters et al., 2000) y un EE de 10 a 23\% (Saunders, 1985); además posee 20 a $25 \%$ de carbohidratos no amiláceos que contienen aproximadamente cantidades iguales de arabinoxilanos y celulosa (Choct, 1997); características que la hacen una fuente energética importante en zonas productoras de arroz, aunque con la limitación relacionada con la alta posibilidad de rancidez de las grasas presentes (Karan, 1998).

La industria avícola genera gran cantidad excretas en las camas; éstas se han usado como alimentos en raciones para rumiantes como fuente de nitrógeno (19 a 32\% proteico y no proteico 48,9 a 54,6\% de la PB total, Kelleher et al., 2002; Mekasha et al., 2002; Chaudhry et al., 1998), minerales, materia seca (MS, 83,1 a $90,1 \%$ ) y materia orgánica (MO, 79-82\%) según Chaudhry et al., 1998; Fontenot, 1979 y Ben-Ghedalia et al., 1996. Cuando se usa la Cp sin procesar, es más barata, pero se corren riesgos sanitarios con patógenos (salmonellas o coccidias, p. ej.).

Este trabajo tuvo como objetivo determinar el efecto de niveles crecientes de Cp en raciones con $\mathrm{Hm}$ o $\mathrm{Pa}$ y bagacillo de caña amonificado ( $\mathrm{Ba}$ ) sobre la digestibilidad de los nutrientes, algunos componentes de la fisiología ruminal y sanguínea de ovinos en crecimiento manejados en confinamiento individual, a fin de establecer las mejores combinaciones de Cp y Hm o Pa que permitan mantener una adecuada salud ruminal y el mayor aprovechamiento de los principales nutrientes en las raciones ofrecidas a estos ovinos.

\section{MATERIAL Y MÉTODOS}

Lugar: Municipio Palavecino, Estado Lara, temperatura media $27^{\circ} \mathrm{C}$, precipitación 846,8 y evaporación $1974,6 \mathrm{~mm}$ anuales, y altura de $550 \mathrm{msnm}$ (datos 1976-2000, estación Tarabana, año 2001). El experimento se realizó desde septiembre de 2001 a marzo de 2002. 
Diseño experimental: Arreglo factorial $4 \times 2$, con 8 tratamientos ( 8 raciones), con un diseño en cuadrado latino $8 \times 8$, una duración de 112 días, se evaluaron cuatro niveles de inclusión (tabla II) de cama de pollos (Cp) con dos fuentes energéticas, $\mathrm{Hm}$ o $\mathrm{Pa}$, en 8 períodos de 14 días, controlando el período y los ovinos, usando 8 corderos de 5 a 6 meses, West African x Dorset, hermanos paternos, con $34,29 \pm 6,59 \mathrm{~kg}$ de peso vivo (PV) o 14,176 $\pm 2,03 \mathrm{~kg}$ de peso metabólico (PM) después del periodo postoperatorio y manejados en confinamiento individual. Se determinó el $\mathrm{pH}$ y nitrógeno amoniacal en licor ruminal, degradabilidad desaparición in vivo de la MS, MO, FDNy FDA (Van Soest, 1994) a las $48 \mathrm{~h}$ de fermentación en el rumen, el consumo individual de alimentos, digestibilidad aparente de la MS y de la MO, así como la digestibilidad de los componentes de la pared celular.

Aleatorización: Ocho ovinos canulados fueron ubicados en corrales al azar, rotando la ración recibida en cada período de modo que cada animal recibía los 8 tratamientos, dividiendo el experimento en dos etapas seleccionando, al azar, el cereal con el que se iniciaría el experimento y ofreciendo primero las cuatro raciones con Hm durante 4 periodos experimentales de 14 días cada uno y luego las otras 4 raciones con $\mathrm{Pa}$ en los últimos 4 períodos en forma sucesiva y aleatoria.

Manejo y mediciones: Los corderos se canularon 28 días antes del inicio del experimento, 12 de una misma edad y peso de mismo origen con la asistencia de un médico veterinario según describe Nouel (2006) y bajo las normas mínimas de bienestar animal durante experimentos, citadas por Zúñiga et al. (2001). Fueron usadas cánulas de látex (BarDiamond $®$ ) con un diámetro interno de $50 \mathrm{~mm}$, un grueso de pared de $6 \mathrm{~mm}$. Los corderos recibieron las 8 raciones en los 8 períodos, 5 días de adaptación, 7 días de medición de consumo ( 8 al 14) y los 3 últimos días (12, 13 y 14) para las mediciones en el rumen. Se recolectaron heces en los días 10 al 14, para hacer digestibilidad con los datos más homogéneos (tres días), manteniendo la confiabilidad necesaria como indican Bertocco et al. (1995); disponían de agua a libertad en cada corral, los comederos eran de $0,5 \mathrm{~m}$ de longitud y capacidad de $25 \mathrm{ly}$ un área de $1,5 \mathrm{~m}^{2}$ para cada animal. El consumo se midió diariamente por diferencia entre lo ofrecido y lo rechazado, manteniendo un $10 \%$ de exceso (consumo ad libitum). El bagacillo de caña molido se amonificó con urea, a razón de $3 \mathrm{~kg}$ por $100 \mathrm{~kg}$ de material, en base a experiencias previas descritas por Hernández y Mújica (2003), con la humedad con la cual llegó de la central azucarera.

Los alimentos y raciones fueron muestreados para determinar la MS, MO (MS menos cenizas.-C.- a $600^{\circ} \mathrm{C}$; AOAC, 1884), PB (nitrógeno total por 6,25, Cioccia et al., 1995), FDN, FDA (Van Soest, 1994), los resultados se presentan en las tablas I y II. Las colecciones de licor ruminal se realizaron antes de ofrecer el alimento ( 0$)$ y a las 3 , 6,9 y 12 horas después de ofrecido, para la medición de $\mathrm{pH}$ se usó directamente un $\mathrm{pH}$ metro portátil al momento de recoger las muestras de licor, conservando las muestras congeladas aplicando la metodología descrita por Preston (1995) a fin de hacer el análisis posterior del nitrógeno amoniacal (Cioccia et al., 1995). La degradabilidad in vivo de MS, PB y FIDN, mediante el uso de bolsas de nylon que fueron incubadas por 48 horas en el rumen según la técnica descrita por Orskov (1982).

Se procedió al análisis de la varianza, separando medias cuando existieron diferencias con la prueba de Tukey, de no tener efecto de covariables, o la de Sheffe al existir covariables significativas (se usó el PV y el PM como covariables en digestibilidad). Los datos tabulados fueron analizados para el modelo propuesto y variables consideradas usando el paquete estadístico Statistix $7.0(2000)$. 


\section{RESULTADOSYDISCUSIÓN}

Las materias primas empleadas ( $\mathrm{Hm}$ y $\mathrm{Pa}$, tabla I) para elaborar las raciones (tabla II) presentan valores similares a los reportados por otros autores; en el caso de los subproductos de cereales tienen valores de 89 a $91 \%$ de MS y 13 a $14 \%$ de PB, similares a los reportados por el NRC (1985) y DePeter et al. (2000) para la Pa y la Hm. Sin embargo, el nivel de $\mathrm{MO}$ y $\mathrm{C}$ es similar para $\mathrm{Hm}$ pero diferente para $\mathrm{Pa}$, sugiriendo una posible contaminación de este producto con cascarilla de arroz. Esto lo corrobora el mayor nivel de fibra presente con respecto a dicho reporte (NRC, 1985). La Cp se corresponde con lo reportado por Rodrígues et al. (1997) para una $\mathrm{Cp}$ de calidad intermedia, pero de inferior calidad proteica y energética a la reportada por Chaudhry et al., 1996.

El consumo de PB, C, FDA y FDN (tabla III) incrementó con el nivel de $\mathrm{Cp}$ en las raciones independientemente del cereal que contenían; mientras que el consumo de MO fue homogéneo para los niveles de cama considerados en las raciones. Esto sugiere que todos los corderos ajustaron su consumo hasta llegar a un nivel más o menos similar de MO o energía en la ración. El consumo de MS para raciones con $\mathrm{Pa}$ fue un $16 \%$ menor al esperado para corderos empleados al compararse con las tablas de nutrientes para ovinos del NRC (1985), donde se prevé un consumo de MS de 1,45 kg para ovinos de $35 \mathrm{~kg}$ de $\mathrm{PV}(4,15 \%)$ pero en el caso de raciones con $\mathrm{Hm}$ fue casi un 9\% superior. El consumo de PB alcanzado para ambos grupos de raciones es superior al requerido para sostener ganancias de peso de 150 a 200 g/animal por día en ovinos de $30 \mathrm{~kg}$ PV (140 a 154 g PB por día) según lo reporta el NRC (1985), y en caso de los mayores niveles de $\mathrm{Cp}$ fue muy superior a esta cifra.

Raposo y Machado (1993) reportan niveles de consumo de 1196 g/cordero/día cuando recibían raciones con $38 \%$ de bagazo de caña prensado al vapor, con $12,37 \%$ de PB y un nivel de sorgo del $34 \%$, esto indica que estos ovinos consumieron unos $147 \mathrm{~g}$ PB por día, de modo que las raciones con Cp y subproductos de cereales pudieron aportar niveles superiores de MS y PB para cubrir las necesidades de los ovinos. Nour et al. (1989) determinaron que raciones con 30 a $50 \%$ de paja de maíz y $11,1 \%$ de PB permitieron altos niveles de consumo de las raciones ofrecidas. Estos consumos fluctuaron entre 1326 y $1331 \mathrm{~g} / \mathrm{animal} /$ día (cerca del 5,1\% de MS del PV), en corderos de 26 $\mathrm{kg}$ de PV y una respuesta animal económicamente sostenible (102 a $127 \mathrm{~g}$ de peso vivo/cordero/día), que es similar a la alcanzada con las raciones de $\mathrm{Cp}$ y Ba evaluadas en este experimento, pero con un nivel de MO ingerida superior y con nivel de PB ligeramente inferior en el caso de ellos.

La digestibilidad aparente de la MS, MO, PB y hemicelulosa (tabla V) fue más elevada en los corderos que recibieron Hm respecto

Tabla I. Composición de las materias primas usadas en la elaboración de las raciones evaluadas. (Composition of the raw materials used in the preparation of evaluated rations).

\begin{tabular}{lcccccc}
\hline Materia prima & MS $\%$ & MO \% & PB \% & EE \% & Cenizas \% FDN \% \\
\hline Harina gruesa de subproductos de maíz & 89,3 & 95,3 & 13,1 & 4,5 & 4,7 & 28,8 \\
Pulitura de arroz & 91,4 & 89,1 & 14,0 & 11,6 & 10,9 & 32,7 \\
Bagacillo de caña amonificado & 65,0 & 82,1 & 9,0 & 0,7 & 17,9 & 85,8 \\
Cama de pollos & 94,4 & 64,2 & 20,6 & 1,1 & 35,9 & 57,3 \\
\hline
\end{tabular}

MS: materia seca; MO: materia orgánica; PB: proteína bruta; EE: extracto etéreo; FDN: fibra insoluble en detergente neutro.

Archivos de zootecnia vol. 60, núm. 229, p. 22. 


\section{USO DE CAMA DE POLLOS, SUBPRODUCTOS DE CEREALES Y CAÑA EN OVINOS}

a Pa. Los corderos con Hm en la ración, tuvieron una digestibilidad de la $\mathrm{PB}$, la $\mathrm{MO}$ y la fibra que no varió sustancialmente o disminuyó a medida que aumentaba la concentración de $\mathrm{Cp}$, la digestibilidad de todos estos componentes, a excepción de la hemicelulosa, aumentó en el caso de $\mathrm{Pa}$. Los resultados obtenidos indican que la degradabilidad in vivo de la MS fue similar a la obtenida con bagazo tratado con hidróxido de sodio (de $51,1 \%$ ) de acuerdo a Gihad et al. (1981). La degradabilidad in vivo obtenida fue superior a la reportada por Thi et al. (2001) para el bagazo sin amonificar, que alcanzó a sólo 25,5, 27,9, y 28,7\% para la MS, MO y FDN respectivamente. Demostrándose la notable mejoría en la fermentación y digestión del Ba y concuerda con los resultados de degradabilidad in vivo de la MS y la MO de fibras largas amonificadas con 1 a $4 \%$ de urea obtenidos por Hadjipanayiotou y Economides (1997). Destacando que la capacidad de fermentación ruminal de este Ba fue mucho menor cuando los corderos consumieron las raciones con $\mathrm{Pa}$. Aun cuando la $\mathrm{Cp}$ es una fuente de nitrógeno no proteico, en la alimentación de rumiantes puede ser útil la presencia de una fuente de nitrógeno, de utilización más inmediata por los microorganismos del rumen, como fue el amoniaco incorporado aquí en el $\mathrm{Ba}$, pudiendo servir de sustrato para la estimulación de estos microorganismos, resultando en una mayor degradabilidad in vivo de la fibra del Ba en el rumen.

De los resultados de degradabilidad in vivo de combinaciones de $\mathrm{Hm}$ con Ba y $\mathrm{Cp}$ obtenidos (tabla VI) se puede apreciar que la degradabilidad in vivo, tanto de la MS como de $\mathrm{MO}$ de $\mathrm{Hm}$, fue considerablemente superior a las del Ba y fue independiente del nivel de Cp ofrecido a los corderos. La degradabilidad in vivo de la FDN mostró una ligera tendencia a incrementarse con el nivel de $\mathrm{Cp}$ en la ración. La máxima degradabilidad in vivo se obtuvo cuando el nivel de Cp fue de 29,4\% que también fue la ración que produjo el mayor $\mathrm{pH}$ del licor ruminal donde los microorganismos celulolíticos actúan con mayor eficiencia. Esto, pudo haber estimulado a los microorganismos afectando positivamente la degradabilidad in vivo de la FDN de Hm.

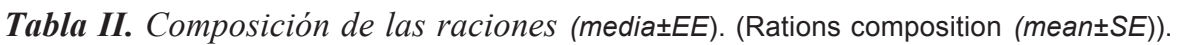

\begin{tabular}{lcccccccc}
\hline Ración $\%$ & 1 & 2 & 3 & 4 & 5 & 6 & 7 & 8 \\
\hline CP & 11,4 & 20,4 & 29,4 & 38,4 & 11,4 & 20,4 & 29,4 & 38,4 \\
HSM & 48 & 39 & 30 & 21 & 0 & 0 & 0 & 0 \\
PA & 0 & 0 & 0 & 0 & 48 & 39 & 30 & 21 \\
BC & 40 & 40 & 40 & 40 & 40 & 40 & 40 & 40 \\
MS & $71,8 \pm 0,5$ & $71,9 \pm 0,2$ & $70,9 \pm 0,3$ & $71,3 \pm 0,2$ & $71,9 \pm 0,1$ & $71,6 \pm 0,3$ & $71,9 \pm 0,4$ & $71,8 \pm 0,1$ \\
PB & $12,3 \pm 0,1$ & $12,7 \pm 0,1$ & $12,9 \pm 0,2$ & $13,7 \pm 0,1$ & $9,7 \pm 0,3$ & $10,6 \pm 0,1$ & $11,5 \pm 0,2$ & $12,4 \pm 0,2$ \\
MO & $59,6 \pm 0,5$ & $57,3 \pm 0,3$ & $55,7 \pm 0,3$ & $50,4 \pm 0,1$ & $55,2 \pm 0,2$ & $51,3 \pm 0,3$ & $48,5 \pm 0,1$ & $46,4 \pm 0,2$ \\
C & $12,2 \pm 0,6$ & $14,6 \pm 0,2$ & $15,2 \pm 0,4$ & $20,9 \pm 0,2$ & $16,7 \pm 0,4$ & $20,3 \pm 0,2$ & $23,4 \pm 0,3$ & $26,1 \pm 0,1$ \\
EE & $2,6 \pm 0,1$ & $2,3 \pm 0,3$ & $2,0 \pm 0,1$ & $1,6 \pm 0,2$ & $5,5 \pm 0,2$ & $4,9 \pm 0,1$ & $3,8 \pm 0,2$ & $3,4 \pm 0,1$ \\
FDN & $60,6 \pm 0,1$ & $61,1 \pm 0,2$ & $65,3 \pm 0,5$ & $66,7 \pm 0,1$ & $52,1 \pm 0,1$ & $55,0 \pm 0,5$ & $56,9 \pm 0,2$ & $61,1 \pm 0,3$ \\
FDA & $37,2 \pm 0,1$ & $40,3 \pm 0,2$ & $42,6 \pm 0,3$ & $48,4 \pm 0,2$ & $38,8 \pm 0,1$ & $43,2 \pm 0,2$ & $46,9 \pm 0,3$ & $50,9 \pm 0,2$ \\
H & $23,4 \pm 0,3$ & $20,7 \pm 0,2$ & $22,8 \pm 0,4$ & $18,3 \pm 0,3$ & $13,3 \pm 0,1$ & $11,8 \pm 0,4$ & $10,0 \pm 0,3$ & $10,1 \pm 0,2$
\end{tabular}

CP: cama de pollos; HSM: harina de subproductos de maíz; PA: pulidura de arroz; BC: bagacillo de caña; MS: materia seca; PB: proteína bruta; MO: materia orgánica; C: cenizas; EE: extracto etéreo; FDN: fibra soluble en detergente neutro; FDA: fibra soluble en detergente ácido. H: hemicelulosa. 
NOUEL BORGES, HEVIA OPAZO, VELÁSQUEZ, ESPEJO DÍAS, ROJAS Y SÁNCHEZ

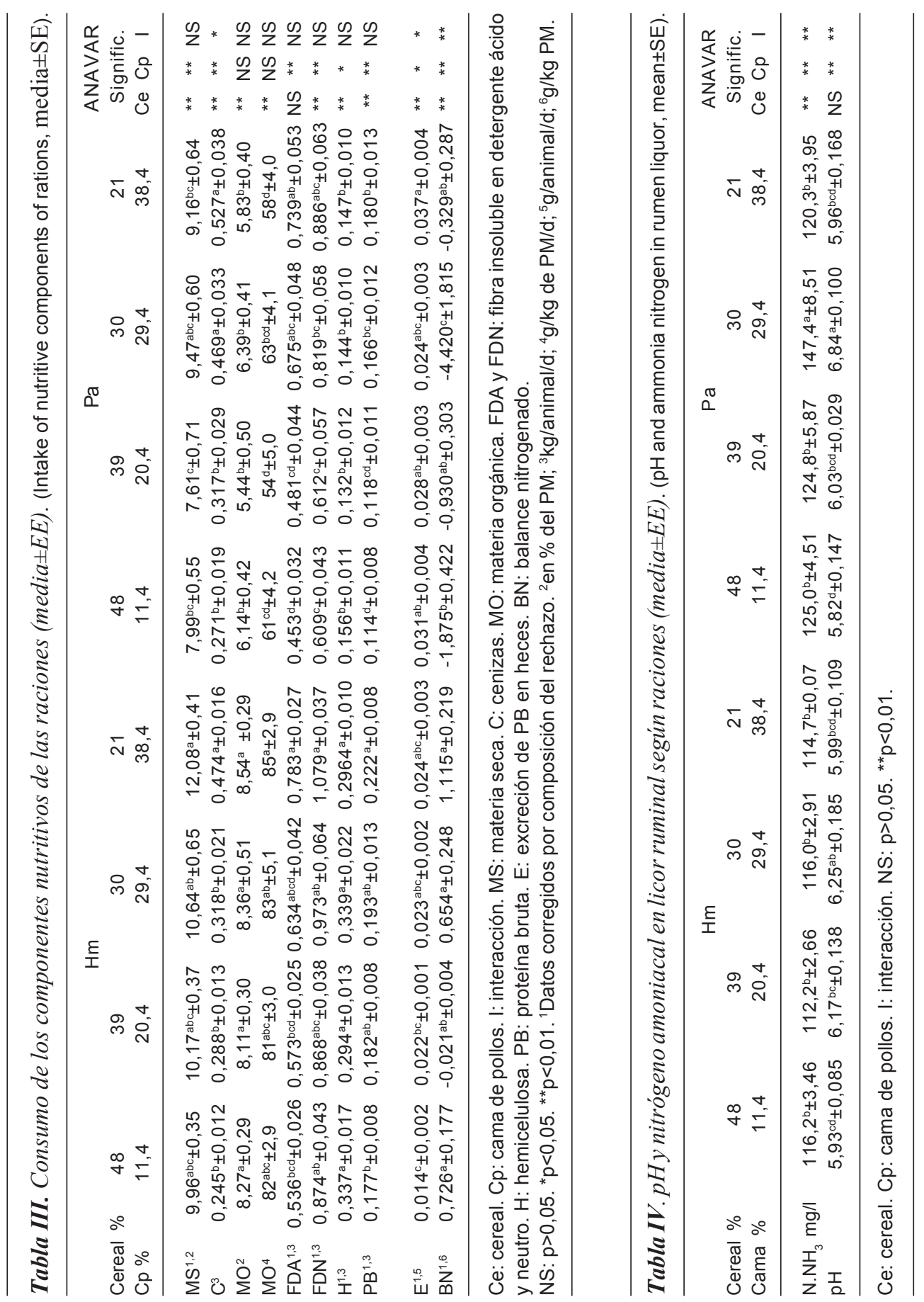

Archivos de zootecnia vol. 60, núm. 229, p. 24. 


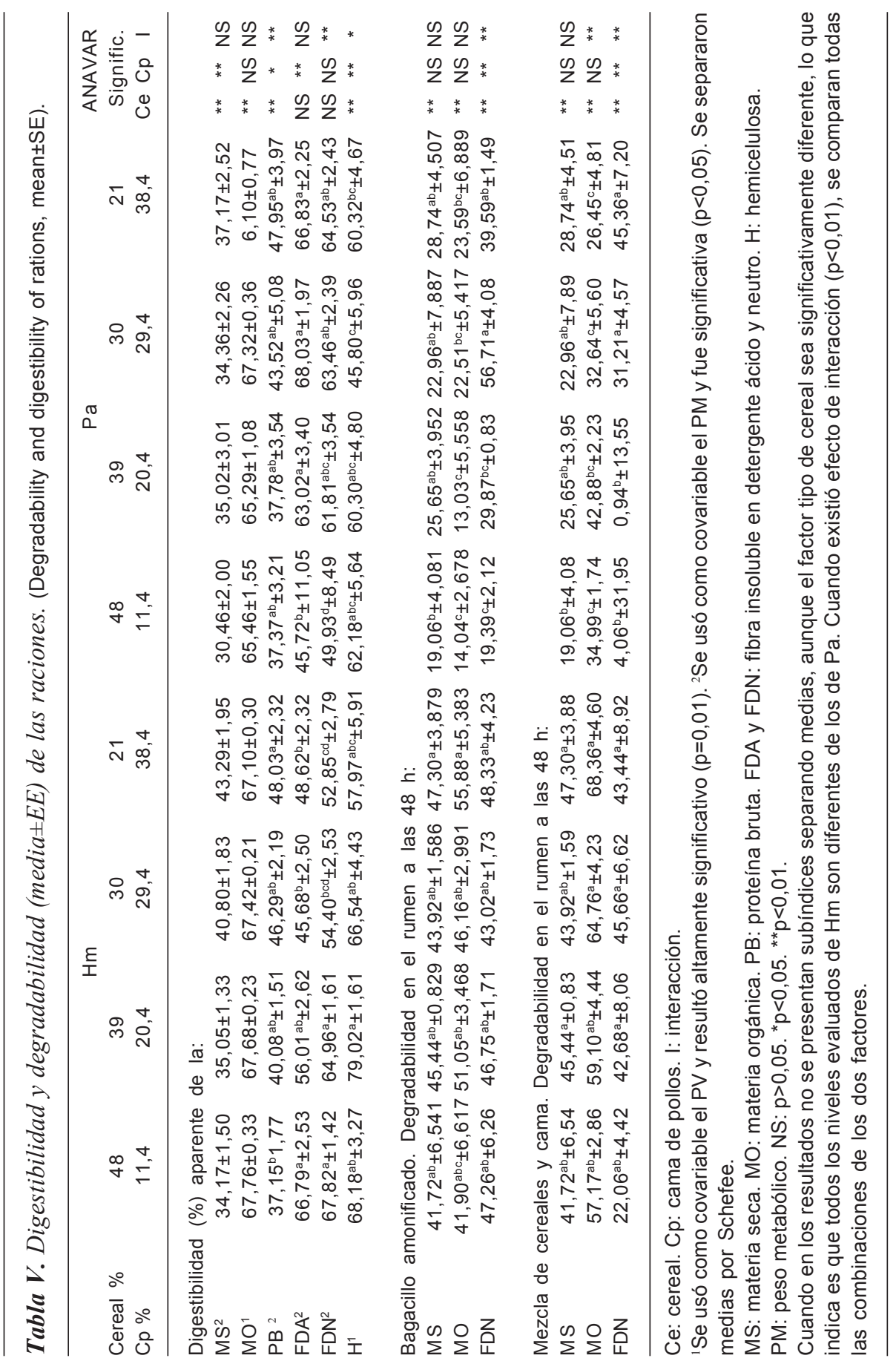

Archivos de zootecnia vol. 60, núm. 229, p. 25. 
NOUEL BORGES, HEVIA OPAZO, VELÁSQUEZ, ESPEJO DÍAS, ROJAS YSÁNCHEZ

Tabla VI. Degradabilidad en el rumen al incubar por 48 h la harina de subproductos de maíz para los niveles de cama evaluados (media $\pm E E$ ). (Rumen degradability at $48 \mathrm{~h}$ incubation of hominy for evaluated poultry litter levels, mean \pm SE).

\begin{tabular}{lccccc}
\hline Nivel de cama \% & 11,4 & 20,4 & 29,4 & 38,4 & Signific. \\
Degradabilidad de la MS & $71,68 \pm 0,50$ & $68,20 \pm 2,25$ & $69,70 \pm 8,54$ & $72,86 \pm 0,78$ & NS \\
Degradabilidad de la MO & $74,65 \pm 1,83$ & $77,79 \pm 7,82$ & $76,65 \pm 6,79$ & $74,66 \pm 0,77$ & NS \\
Degradabilidad de la FDN & $19,33 \pm 1,28$ & $21,28 \pm 4,40$ & $33,07 \pm 12,78$ & $26,44 \pm 4,60$ & NS \\
\hline
\end{tabular}

NS: $p>0,05$.

La MS, MO o FDN presentes en el Ba, cereales y $\mathrm{Cp}$ de las raciones, mostraron un grado de degradabilidad in vivo mayor en las raciones con $\mathrm{Hm}$ que con $\mathrm{Pa}$. La degradabilidad in vivo de la MS y MO de mezclas de $\mathrm{Hm}$ y $\mathrm{Cp}$ o Pa y $\mathrm{Cp}$ fue, en general, mayor que la del $\mathrm{Ba}$; todas ellas (Hm y $\mathrm{Cp}$ o Pa y Cp o Ba) sustancialmente menores que las degradabilidad in vivo determinadas para la Hm. También se puede observar que para la FDN, la materia prima más degradable fue la del Ba y la menos degradable fue la presente en Hm. Esto se cumplió en las raciones con $\mathrm{Hm}$, ya que la degradabilidad in vivo de la FDN en el rumen de los que consumían $\mathrm{Pa}$ con los niveles más bajos de $\mathrm{Cp}$ fue muy baja. La baja degradabilidad in vivo de la FIDN, presente en la ración, que se observó en los corderos que consumían Pa junto con niveles bajos de $\mathrm{Cp}$, destaca debido a que la degradabilidad in vivo de la misma fibra en el $\mathrm{Ba}$, medida en los mismos corderos fue más alta. Esto sugiere que en la ración, había algún componente que interfirió con la degradabilidad in vivo de la fibra en la ración y que no está presente en el $\mathrm{Ba}$. Comparando la degradabilidad in vivo y las digestibilidades in vivo de las raciones (tabla V) se puede apreciar que la interferencia de la $\mathrm{Pa}$ en la degradabilidad in vivo, también afectó la digestibilidad de las mismas ya que las digestibilidades de la MS, MO, PB así como de FDN o FDA medidas en los corderos que consumieron $\mathrm{Pa}$, mejoraron al aumentar los niveles de $\mathrm{Cp}$, o igual, reduciendo los niveles de $\mathrm{Pa}$.

Los contenidos de nitrógeno amoniacal (tabla IV) fueron mayores en los corderos que consumieron Pa que en los que consumieron $\mathrm{Hm}$. El efecto del nivel de $\mathrm{Cp}$, sobre el contenido de nitrógeno amoniacal, fue diferente de acuerdo al cereal. Así, en el caso de los corderos que consumieron $\mathrm{Hm}$, los niveles de $\mathrm{Cp}$ tuvieron muy poco efecto sobre el nitrógeno amoniacal mientras que en los asignados a $\mathrm{Pa}$, el nivel de $\mathrm{Cp}$ si afectó el contenido de este tipo de nitrógeno. Esto se detectó principalmente en los corderos asignados a la ración con 29,4\% de Cp. Así, en el licor ruminal de estos corderos el nitrógeno amoniacal fue sustancialmente mayor que los asignados a concentraciones mayores o menores de Cp. Un aumento en el nitrógeno amoniacal en el licor ruminal, se puede interpretar como una reducción en la capacidad de las bacterias del rumen en incorporar nitrógeno en nuevas proteínas. En consecuencia, este nitrógeno se pierde en heces y orina. Esto explicaría las más altas excreciones de nitrógeno en los corderos que consumieron las raciones con $\mathrm{Pa}$ (tabla III) así como con la observación que el balance nitrogenado más negativo de todo el experimento, estuvo asociado con el consumo de la ración que contenía $30 \%$ de Pa y $29,4 \%$ de Cp que, también fue el grupo con la más alta concentración de nitrógeno amoniacal en el licor ruminal.

Se observa que el nivel de Cp tuvo un efecto en el pH del licor ruminal (tabla IV). Tanto en los que consumieron $\mathrm{Hm}$ como $\mathrm{Pa}$, el pH se hizo progresivamente más alcalino al aumentar el nivel de $\mathrm{Cp}$ en la ración. Esta tendencia ascendente se observó sólo has-

Archivos de zootecnia vol. 60, núm. 229, p. 26. 
ta una concentración de Cp de 29,4\% ya que a partir de esta concentración el pH disminuyó. Se aprecia que el cereal consumido modificó el efecto del nivel de Cp. Así, la tendencia ascendente descrita, fue tenue en el caso de los corderos que consumieron la $\mathrm{Cp}$ con $\mathrm{Hm}$ y más pronunciada con $\mathrm{Pa}$. De acuerdo con esto, el diferencial de $\mathrm{pH}$ en el primer caso fue de 0,32 mientras que en el segundo fue de 1,02 unidades de $\mathrm{pH}$. Estas variaciones en el $\mathrm{pH}$ del licor ruminal que ocurrieron en respuesta al consumo de $\mathrm{Cp}$, coincidieron con las variaciones observadas en el contenido de nitrógeno amoniacal. Los corderos que consumieron $\mathrm{Pa}$, fueron los que mostraron mayor variabilidad tanto en $\mathrm{pH}$ como en contenido de nitrógeno amoniacal, el coeficiente de correlación entre estas variables fue $r=0,82$. Indicando que la incorporación de $\mathrm{Pa}$ en la ración estuvo asociada a mayores cambios en el contenido de nitrógeno amoniacal y en el pH que la incorporación de $\mathrm{Hm}$. Esto, en conjunto con la menor granulometría y la alta producción de espuma asociada con el consumo de $\mathrm{Pa}$, posiblemente contribuyeron a la menor digestibilidad de la MS, MO y PB detectada in vivo en los corderos que consumieron este cereal.

Las raciones con Pa tenían un elevado contenido de cenizas (principalmente de la $\mathrm{Cp}$ y $\mathrm{Pa}$ ), además de cantidades significativas de extracto etéreo, en conjunto con una fina granulometría y la temperatura ruminal, pudo favorecer la saponificación de grasas, generando jabón que pudo incrementar la producción de espuma observada en los ovinos que recibieron $\mathrm{Cp}$ y $\mathrm{Pa}$ en sus raciones, la cual probablemente no pudo ser reducida al tener una ración finamente molida que inhibió o redujo la producción de saliva debido a una rumia reducida. Todo esto generó un ambiente favorable para una baja degradación de la fibra y demás componentes de las raciones con $\mathrm{Cp}$ y $\mathrm{Pa}$.

Glock y Degroot (1998) indican que un pH entre 5,0 y 5,2 en licor ruminal, se considera como indicio de acidosis e incluso un
pH de 5,5 puede estar asociado con la proliferación de bacterias ácido lácticas, precursoras de ruminitis. Asimismo, en los casos de intoxicación por consumo elevado de urea, los niveles de nitrógeno amoniacal y $\mathrm{pH}$, medidos en el licor ruminal post mórtem, varían entre 300 y 800 ppm y entre 7,7 y 8,7 respectivamente (Ortolani et al., 2000), indicando que estas condiciones del rumen, son incompatibles con la vida de los rumiantes. Analizando las condiciones del rumen, se aprecia que los valores de $\mathrm{pH}$ y nitrógeno amoniacal obtenidos, no alcanzaron los considerados como indicativos de acidosis ni de intoxicación con amoniaco. Sobre la base que el nitrógeno no proteico de la $\mathrm{Cp}$ es principalmente ácido úrico y la velocidad de conversión de este ácido a amoniaco en el rumen es menor que la de la urea (Oltijen et al., 1968). Esto probablemente protegió a los corderos y debe considerarse como una ventaja adicional del uso de $\mathrm{Cp}$ con relación a otras formas de nitrógeno no proteico. Se observa que (tabla V) independientemente del nivel de $\mathrm{Cp}$, la capacidad de degradar la MS, MO o la FIDN presentes en el $\mathrm{Ba}$ fue superior en corderos que consumían Hm que en los que consumían Pa. También muestra que en el caso de los corderos que consumían $\mathrm{Pa}$, la degradabilidad in vivo de la fibra fue menor en las raciones con menos $\mathrm{Cp}$. Llama la atención el pico de degradabilidad in vivo de la fibra presente en el $\mathrm{Ba}$, en los corderos que consumieron $\mathrm{Pa}$ al $30 \%$ y $\mathrm{Cp}$ al $29,4 \%$. El consumo de esta ración correspondió a un $\mathrm{pH}$ en el licor ruminal de 6,03, probablemente el más favorable para la acción celulítica de las bacterias del rumen. Sin embargo, el consumo de esta ración estuvo asociada con la menor digestibilidad de la hemicelulosa presente en la ración en los estudios in vivo (tabla $\mathbf{V}$ ) indicando que las modificaciones en la disponibilidad de los nutrientes presentes en las raciones con $\mathrm{Pa}$ son complejas.

El efecto negativo de $\mathrm{Pa}$ sobre la digestibilidad y degradabilidad in vivo de la 
ración consumida no se corresponde con las condiciones de $\mathrm{pH}$ y nitrógeno amoniacal del licor ruminal, ya que estas estuvieron dentro de los rangos aceptables. Murguía et al. (2003) reportan, que incluso en ovinos con signos de acidosis y ruminitis, asociados a consumos de granos de maíz, melaza y $\mathrm{Cp}$, la digestibilidad de los componentes de la ración se mantuvo dentro de niveles normales. Reforzando la idea donde los efectos de Pa sobre la función ruminal, se asocia más a limitaciones en condiciones físicas y químicas del rumen, como dispersabilidad de los componentes de la ración en el licor ruminal y la posibilidad de liberar los gases producidos. Esto puede estar asociado con la granulometría pulverulenta (diámetro de partículas menores de $1 \mathrm{~mm}$ ) de la $\mathrm{Pa}$, como ya se indicó es un polvo muy fino, que en conjunto con la grasa de la Pa y los elevados contenidos de minerales de $\mathrm{Pa}$ y de la $\mathrm{Cp}$, pudieron formar una sustancia jabonosa, la cual no pudo ser reducida por la saliva al presentarse poca rumia debido al tamaño de partícula reducido del Ba, que pudo limitar el proceso clave en la eliminación de gases y control del pH ruminal.

\section{CONCLUSIONES}

Los corderos que consumieron niveles

\section{BIBLIOGRAFÍA}

AOAC. 1984. Official methods of analysis (14 ed.). Association of Official Agricultural Chemists. Washington.

Ben-Ghedalia, D., Yosef, E., Miron, J. and Est, Y. 1996. Apparent digestibility of minerals by lactating cows from a total mixed ration supplemented with poultry litter. J. Dairy Sci., 79: 454-458.

Bertocco, J., Moraes, A. e Dal Secco, M. 1995. Efeito do periodo de colecta sobre a digestibilidade de algums nutrientes, em ensaios com ovinos. Rev. Soc. Bras. Zootecn., 24: 261.

Chaudhry, S., Fontenot, J., Naseer, Z. and Ali, C. 1996. Nutritive value of deep stacked and ensiled crecientes de $\mathrm{Cp}$ con $\mathrm{Ba}$ y $\mathrm{Hm}$ mostraron consumo de alimento (10,0 a 12,1\% del PM en MS), digestibilidad (34,2 a 43\% DMS, 67,8 a $67,1 \%$ DMO y 66,8 a 48,6\% DFDA), así como condiciones del licor ruminal y capacidad del rumen para degradar los componentes de la ración, superando a las raciones con $\mathrm{Cp}$, Ba y $\mathrm{Pa}$.

Cuando las mezclas con $\mathrm{Cp}$, Ba y $\mathrm{Pa}$ se ofrecieron a los corderos consumieron y digirieron la ración en menor magnitud (30,5 a $37,2 \%$ DMS, 65,5 a $66,1 \%$ DMO y 45,7 a $66,8 \%$ de DFDA) y baja capacidad para degradar los componentes de la ración en el rumen que raciones con $\mathrm{Cp}$, Ba y $\mathrm{Hm}$.

La incorporación de niveles crecientes de $\mathrm{Cp}$ en raciones con $\mathrm{Hm}$, favoreció mayor consumo (1,44 y 1,42 kg MS/cordero/día) de las R evaluadas que con $\mathrm{Cp}$ y $\mathrm{Pa}$, permitiendo establecer que a menores niveles de $\mathrm{Cp}$ $(11,4$ y $20,4 \%)$ se alcanzaron la mayor digestibilidad de los nutrientes.

\section{AGRADECIMIENTOS}

Al Consejo de Desarrollo Científico Humanístico y Tecnológico de la UCLA por haber financiado este proyecto en su totalidad (023-AG-2000); a la Unidad de Investigación en Producción Animal de la UCLA y al laboratorio de Nutrición de la USB por su apoyo.

broiler litter for sheep. Anim. Feed Sci. Tech., 57: 165-173.

Chaudhry, S., Fontenot, J. and Naseer, Z. 1998. Effect of deep stacking and ensiling broiler litter on chemical composition and pathogenic organisms. Anim. Feed Sci. Tech., 74: 155-167.

Choct, M. 1997. Feed non-starch polysaccharides: Chemical structures and nutritional significance. Feed Milling International (June issue): 13-26. Cioccia, A., González, E., Pérez, M., Mora, J., Romer, H., Molina, E. and Hevia, P. 1995. Application of a colorimetric method to the determination of the protein content of comercial foods, mixed human diets and nitrogen

Archivos de zootecnia vol. 60, núm. 229, p. 28. 


\section{USO DE CAMA DE POLLOS, SUBPRODUCTOS DE CEREALES Y CAÑA EN OVINOS}

losses in infantile diarrhea. J. Food Sci. Nutr., 46: 21-29.

DePeters, E., Fadel, J., Arana, M., Ohanesian, N.P., Etchebarne, M., Hamilton, C., Hinders, R., Maloney, M., Old, C., Riordan, T., Pérez-Monti, H. and Pareas, J. 2000. Variability in the chemical composition of seventeen selected by-product feedstuffs used by the California dairy industry. Prof. Anim. Scient., 15: 1-31.

Fontenot, J. 1979. Alternatives in animal waste utilization. Introductory comments. J. Anim. Sci., 48: 111-112.

García, G. 1980. Mejoramiento nutricional con bagazo de caña, en ovejos (mimeo). Centro de Investigaciones Agropecuarias Región Centroccidental. Ministerio de Agricultura y Cría. El Cují. Barquisimeto. $18 \mathrm{pp}$.

Gihad, E., El-Bedawy, T. and Allam, S. 1981. Comparative efficient of utilization of untreated and $\mathrm{NaOH}$-treated poor quality roughages through in situ digestion by sheep, goats and buffaloes. International Symposium on Nutrition and Systems of Goat Feeding. Vol. I. Tours. France.

Glock, R. and Degroot, B. 1998. Suden death in feedlot cattle. J. Anim. Sci., 76: 315-319.

Hadjipanayiotou, M. and Economides, S. 1997. Assessment of various treatment conditions affecting the ammoniation of long straw by urea. Livest. Res Rural Dev., 9: 5.

Hernández, A. y Mújica, A. 2003. Optimización del proceso de amonificación en seco y valor nutritivo del bagazo de caña ofrecido a ovinos. Trabajo Especial de Grado. Universidad Nacional Experimental Politécnica Antonio José de Sucre. Barquisimeto. 150 pp.

Hernández, B., Guerra, M. and Rivero, F. 1999. Effect of the fractioning on the characteristics of the deffated corn germ. Cienc. Tecnol. Aliment., 19: 107-112.

Karan, A. 1998. Separation of oryzanol from crude rice bran oil. Thesis for the degree of Master of Applied Science Graduate. Department of Chemical Engineering. University of Toronto. Toronto. Canada. 122 pp.

Kelleher, B., Leahy, J., Henihan, A., O'Dwyer, T., Sutton, D. and Leahy, M. 2002. Advances in poultry litter disposal technology - a review. Bioresource Technol., 83: 27-36.

MAC. 1998. Anuario estadístico agropecuario 1996.
Dirección General Sectorial de Planificación y Políticas. Dirección de Estadísticas e Informática. Caracas. 307 pp.

Mekasha, Y., Teegegne, A., Yami, A. and Umuna, N. 2002. Evaluation of non-conventional agroindustrial by-products as supplementary feeds for ruminants: in vitro and metabolism study with sheep. Small Ruminant Res., 44: 25-35.

Murguía, M., Pacheco, J. y Castellanos, A. 2003. La rumenitis causada por acidosis ruminal no afecta a la digestibilidad de los nutrimentos en ovinos Pelibuey. Tecnología Pecuaria Méxicana, 41: 329-336.

NRC. 1985. Nutrient requirements of sheep. $6^{\text {th }}$ revised edition. National Academy Press. Washington DC. 92 pp.

Nouel, G. 2006. Uso de cama de pollo y subproductos de maíz o arroz y caña en la alimentación de ovinos confinados. Tesis Doctoral. Programa Doctorado en Nutrición. Universidad Simón Bolívar. Sartenejas Estado Miranda. $133 \mathrm{pp}$.

Nour, A., Abou Akkada, A., Nour, A. and Mabrouka, A. 1989. The optimum level of roughages in the diets of sheep. In: Overcoming constraints to the efficient utilization of agricultural by-products as animal feed. Proceedings o the $4^{\text {th }}$ Workshop held at the Institute of Animal Research. Mankon Station. Ed. A. Said and B. Dzowela. Bamenda. Cameroon. ARNAB. Addis Ababa. Ethiopia.

Oltijen, R., Shyter, L., Kozok, A. and Williams, J. 1968. Evaluation of urea, biurate, urea phosphate and eric acid as NPN sources for cattle. J. Anim. Nutr., 94: 193-202.

Orskov, E. 1982. Protein nutrition in ruminants. Academic Press. London. pp. 47-55.

Ortolani, E., Brito, L., Schalch, U., Pacheco, J. and Baldacci, L. 1997. Botulism outbreak associated with poultry litter consumption in three Brazilian cattle herds. Vet. Hum. Toxicol., 39: 89-92.

Ortolani, E., Mori, C. and Rodríguez-Filho, J. 2000. Ammonia toxicity from urea in a Brazilian dairy goat flock. Vet. Hum. Toxicol., 42: 87-89.

Preston, T. 1995. Tropical animal feeding. Paper \# 126. FAO. Roma.

Ramírez, V. 2000. Análisis del circuito azucarero en Venezuela. Primer Encuentro de Porcicultores de la Región Centroccidental San Felipe Yaracuy. UNEY. Venezuela. 


\section{NOUEL BORGES, HEVIA OPAZO, VELÁSQUEZ, ESPEJO DÍAS, ROJASY SÁNCHEZ}

Raposo, S. and Machado, P. 1993. Effect of the replacement of steam treated sugar cane bagasse by milo on ruminal fermentation in bovines and in vivo digestibility in sheep. Livest. Res. Rural Dev., 5(2). http://www.Irrd.org/ Irrd5/2/brazil1.htm (02/02/09)

Rodrígues, A., Da Cruz, G. e Esteves, S. 1997. Utilização de cama de frango na alimentação de bovinos. Embrapa-CPPSE. Circular Técnica 10. São Carlos. 28 pp.

Saunders, R. 1985. Rice bran: composition and potential food uses. Food Reviews International, 1: $465-495$.

Statistix 7.0. 2000. By Analytical Software.
Tallahassee. Florida. www.statistix.com Thi, N., Duc, D. and Linh, L. 2001. The chemical composition and in sacco degradability of some bedding materials from agricultural by - products (rice straw, sugar cane bagasse, rice husk). Proceeding Workshop on Improved Utilization of By-products for Animal Feeding in Vietnam. NUFU project-3/. Hanoi.

Van Soest, P. 1994. Nutritional ecology of the ruminant. $2^{\circ}$ edition. Univ. Cornell Press. Ithaca. Zúñiga, J., Tur-Marí, J., Milocco, S. y Piñeiro, R. 2001. Ciencia y tecnología en protección y experimentación animal. McGraw Hill Interamericana. Madrid. 682 pp.

Archivos de zootecnia vol. 60, núm. 229, p. 30. 